\title{
Fully Three-Dimensional Bioprinted Skin Equivalent Constructs with Validated Morphology and Barrier Function
}

\author{
Kristy Derr, BS, Jinyun Zou, MS, ${ }^{2}$ Keren Luo, BS, ${ }^{2}$ Min Jae Song, PhD, ${ }^{3}$ G. Sitta Sittampalam, PhD, \\ Chao Zhou, PhD, ${ }^{2,4}$ Sam Michael, BS, ${ }^{1}$ Marc Ferrer, PhD, ${ }^{1}$ and Paige Derr, PhD ${ }^{1}$
}

Development of high-throughput, reproducible, three-dimensional (3D) bioprinted skin equivalents (BPSEs) that are morphologically and functionally comparable to native skin tissue is advancing research in skin diseases, and providing a physiologically relevant platform for the development of therapeutics, transplants for regenerative medicine, and testing of skin products like cosmetics. Current protocols for the production of engineered skin grafts are limited in their ability to control 3D geometry of the structure and contraction leading to variability of skin function between constructs. In this study, we describe a method for the biofabrication of skin equivalents (SEs) that are fully bioprinted using an open-market bioprinter, made with commercially available primary cells and natural hydrogels. The unique hydrogel formulation allows for the production of a human-like SE with minimal lateral tissue contraction in a multiwell plate format, thus making them suitable for high-throughput bioprinting in a single print with fast print and relatively short incubation times. The morphology and barrier function of the fully 3D BPSEs are validated by immunohistochemistry staining, optical coherence tomography, and permeation assays.

Keywords: bioprinting, skin, barrier function, keratinocytes, high-throughput screening

\section{Impact Statement}

This article describes a method for the biofabrication of skin tissue equivalents in a multiwell plate format. The technique and results overcome shortcomings of previously published engineering methods, and show good architecture and barrier function from well to well; thus it may be used for compound functional testing and for the development of disease tissue models for screening.

\section{Introduction}

$\mathbf{T}$ HREE-DIMENSIONAL (3D) BIOPRINTING Is an emerging bioengineering technique that offers great opportunity to expand output and improve precision of traditional tissue engineering methods. ${ }^{1}$ Three-dimensional bioprinting facilitates the creation of tissues in a layer-by-layer approach, creating deliberate cell patterns while preserving cell function and viability within the printed construct. Methods of deposition include pneumatic or plunger-facilitated direct dispenser, light-assisted printing, and contactless jetting dispenser. ${ }^{2}$ The technique provides unique capabilities that the basic pipetting protocol cannot achieve in that it is highly automatable, reproducible, readily adaptable to a high throughput multiwell plate format, provides more control over geometry of the construct, and enables tissue structures to be built without the need for a well wall or side support along the edges. In addition to the potential of $3 \mathrm{D}$ bioprinting to increase reproducibility and scalability of the biofabrication of tissues such as dermal tissue, it should enable the future construction of more complex skin architecture such as vasculature, hair follicles, rete ridges, and nerve endings.

Skin is the largest organ of the body and provides protection from the environment, microbes, parasites, heat, UV rays, and water loss. It is a highly complex organ that acts as a barrier from external substances. ${ }^{3-5}$ The skin is composed

\footnotetext{
${ }^{1}$ Department of Preclinical Innovation, National Center for Advancing Translational Sciences, National Institutes of Health, Rockville, Maryland

${ }^{2}$ Department of Electrical and Computer Engineering, Bethlehem, Pennsylvania.

${ }^{3}$ National Eye Institute, National Institutes of Health, Rockville, Maryland.

${ }^{4}$ Department of Bioengineering, Lehigh University, Bethlehem, Pennsylvania.
}

(C) Kristy Derr et al. 2019; Published by Mary Ann Liebert, Inc. This Open Access article is distributed under the terms of the Creative Commons Attribution Noncommercial License (http://creativecommons.org/licenses/by-nc/4.0/) which permits any noncommercial use, distribution, and reproduction in any medium, provided the original author(s) and the source are cited. 
of three layers: the fatty hypodermis, the dermis containing fibroblasts embedded in a high concentration of extracellular matrix (ECM) with other structures such as capillaries and hair follicles, and the multistratified epidermis composed primarily of keratinocytes in varying stages of differentiation. Within the epidermis, the stratum basale, stratum spinosum, and the stratum granulosum make up the main live layers that proliferate from the basal layer yielding the terminally differentiated corneocytes in the stratum corneum. ${ }^{6}$

In vitro human skin models are being developed as transplants for regenerative medicine and for toxicity testing of skin products and therapeutics in place of animal testing experiments such as dermal absorption or corrosivity studies. $^{7,8}$ In fact, EU regulations (Regulation [EC] No. 1223/ 2009) mandate that skin products cannot be tested on animals and must be evaluated using alternative skin models. Excised skin from human donors has been used for ex vivo testing of compounds but presents challenges in availability and variability. ${ }^{9}$ As such, there has been a push toward the development of basic engineered skin equivalents (SEs) with significant success. ${ }^{10,11}$ To build upon these successes, 3D bioprinting has the potential to increase reproducibility, scalability, and build more complex architecture.

There have been notable efforts to generate bioprinted SEs (BPSEs) in recent years, although several obstacles still remain. Techniques include pneumatic direct dispense, microvalve extrusion, nebulizing, laser-assisted bioprinting, and convergent trifurcated syringes with a variety of bioinks incorporating such materials as collagen, alginate, fibrin, gelatin, and Polyvinylpyrrolidone ${ }^{12-16}$ Recent studies have presented skin that is only partially bioprinted, or printed in phases around incubation steps. ${ }^{12-15}$ Some published protocols require a long print time up to $40 \mathrm{~min} / \mathrm{tissue}^{14,16}$ or maturation time up to $26-35$ days. ${ }^{13,15}$ Oftentimes such studies utilize a custom-built bioprinter with complicated printing processes. ${ }^{12,14,16}$ Additionally, in many cases validation studies lack evidence of human skin like function or morphology for in vitro SEs. ${ }^{12,13,16,17}$ The purpose of this study was to develop a biofabricated SE that is fully printed on an open-market printer in a single protocol using commercially available primary cells, having human skin-like morphology and validated barrier function. Lateral contraction is minimized, making the protocol suitable for miniaturization and high-throughput production for compound testing.

\section{Materials and Methods}

\section{Cell culture}

Neonatal human dermal fibroblasts $\left(\mathrm{HDF}_{\mathrm{N}}\right.$, Zen Bio DFN-F) and Neonatal Normal Human Epithelial Keratinocytes $\left(\mathrm{NHEK}_{\mathrm{N}}\right.$, ATCC PCS-200-010 [for experiments in 12well format] or ScienCell 2100 [for experiments in 24-well format]) were cultured at $37^{\circ} \mathrm{C}, 5 \% \mathrm{CO}_{2}$ in Dulbecco's modified Eagle's medium (Gibco 11965), 10\% Fetal Bovine Serum (HyClone), 1\% Penicillin-Streptomycin (Gibco). $\mathrm{NHEK}_{\mathrm{N}}$ were cultured at $37^{\circ} \mathrm{C}, 5 \% \mathrm{CO}_{2}$ in Keratinocyte Media (Lonza 192060). $\mathrm{HDF}_{\mathrm{N}}$ and $\mathrm{NHEK}_{\mathrm{N}}$ were frozen in aliquots at passage 5 and 3, respectively. Aliquots of each were thawed before printing and harvested for print at 60-80\% confluency.

\section{Dermal base hydrogel preparation}

Gelatin (Sigma G1890, final concentration $0.045 \mathrm{mg} / \mathrm{mL}$ ) was dissolved into fibrinogen stock solution $(20 \mathrm{mg} / \mathrm{mL}$ fibrinogen dissolved in $0.0087 \mathrm{mg} / \mathrm{mL} \mathrm{NaCl}$ ) (Sigma F3879, final concentration in dermal base hydrogel (DBH) mixture is $7.7 \mathrm{mg} /$ $\mathrm{mL})$ at $37^{\circ} \mathrm{C}$. After complete dissolution of gelatin, collagen I solution (Corning 354249, final concentration $4 \mathrm{mg} / \mathrm{mL}$ ) was added and mixed thoroughly. The mixture was brought to $37^{\circ} \mathrm{C}$, at which time Elastin was added (Spectrum E1217, final concentration $0.55 \% \mathrm{v} / \mathrm{v})$. A $10 \times$ phosphate-buffered saline (PBS) solution was added to balance the collagen solution, and water to bring the mixture to total volume. The mixture was kept at $37^{\circ} \mathrm{C}$ before use and neutralized with $1 \mathrm{~N} \mathrm{NaOH}$ immediately before adding $\mathrm{HDF}_{\mathrm{N}}$.

\section{Syringe preparation}

A three-syringe configuration was used: one plunger, one jetting dispenser and one direct dispenser.

Syringe 1, dermis. $\mathrm{HDF}_{\mathrm{N}}$ were harvested using $0.05 \%$ Trypsin (Gibco), pelleted, and resuspended in prepared DBH at a concentration of 2 million/mL. The molten DBH/ cell mixture was loaded into a plunger syringe (regenHU 900013152) and briefly chilled on ice to gel. After attaching a $0.41 \mathrm{~mm}$ ID luer lock needle (Nordson 7018263), the syringe was loaded onto a regenHU 3DDiscovery Bioprinter. Volumetric plunger dispensing for the DBH mixture provides an advantage over pneumatic in that the dispense rate is not notably affected by small variation in viscosity with each gel preparation.

Syringe 2, basal layer. Laminin/Entactin (Corning $354259,1.61 \mathrm{mg} / \mathrm{mL}$ in Gibco DMEM 11965), was loaded into a chilled $\left(5^{\circ} \mathrm{C}\right)$ syringe (Nordson 7012072$)$ on the regenHU 3DDiscovery with jetting dispenser to facilitate application of a thin uniform layer.

Syringe 3, epidermis. NHEK $_{N}$ were harvested with $0.05 \%$ Trypsin, pelleted and resuspended at a concentration of 6.15 million cells $/ \mathrm{mL}$ in Keratinocyte Media. Cells were pipetted into a syringe (Nordson 7012072) fitted with a $0.10 \mathrm{~mm}$ ID needle (Nordson 7018477) with pneumatic extrusion, which enabled cell agitator (regenHU 900007250) to be attached.

\section{D printing of tissue construct}

The schema for optimized BPSEs is shown in Figure 1. The architecture was designed using regenHU BioCAD ${ }^{\mathrm{TM}}$ Software. The dermal level was extruded from syringe 1 in 6 circular layers $\sim 0.4 \mathrm{~mm}$ thick with a diameter of $11.4 \mathrm{~mm}$, directly onto the membrane of the transwell insert (Corning 3460) using a plunger dispenser. Immediately afterward, syringe 2 evenly coated the top of the tissue with $7.5 \mu \mathrm{L}$ laminin/entactin with the jetting dispenser. Syringe 3 then deposited $26 \mu \mathrm{L} \mathrm{NHEK}_{\mathrm{N}}$ suspension onto the center of the tissue construct using the direct dispenser. To facilitate fibrinogen-fibrin conversion, $300 \mu \mathrm{L}$ keratinocyte media containing thrombin (5 NIH unit/mL; Sigma T6884) mixture was placed in each well, beneath the transwell insert. 
a

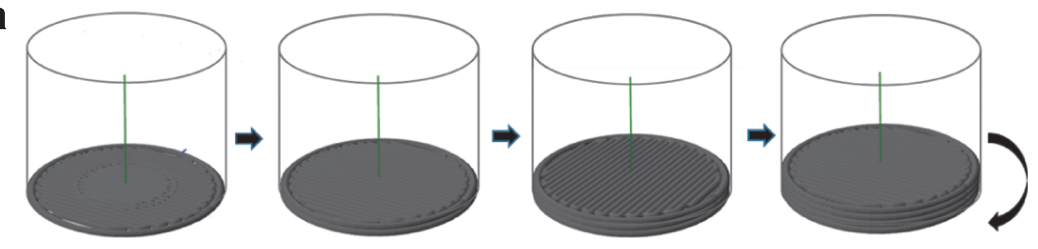

FIG. 1. Schematic of printed construct. (a) Three levels comprise the structure: dermis, laminin/entactin basal layer, and epidermis. (b) Incubation method after printing. BPSEs are incubated for $1.5 \mathrm{~h}$ at RT with thrombin/ media placed underneath the transwell.

Samples are subsequently submerged in a medium and placed in incubator for 1 week. On day 7 medium is lowered and tissues are incubated at air-liquid interface for 1 week. BPSEs, bioprinted skin equivalent.

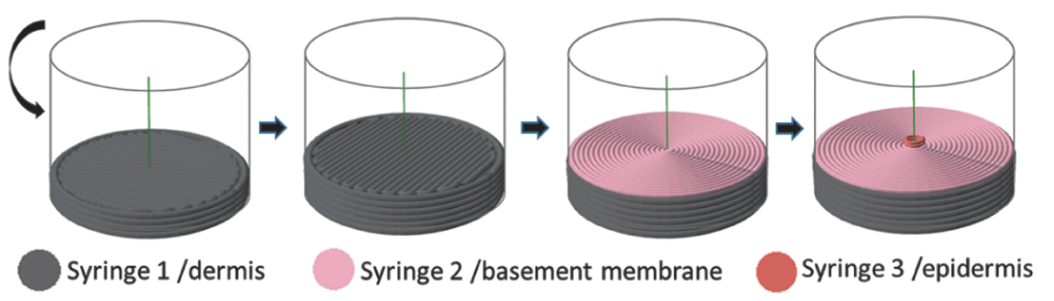

b

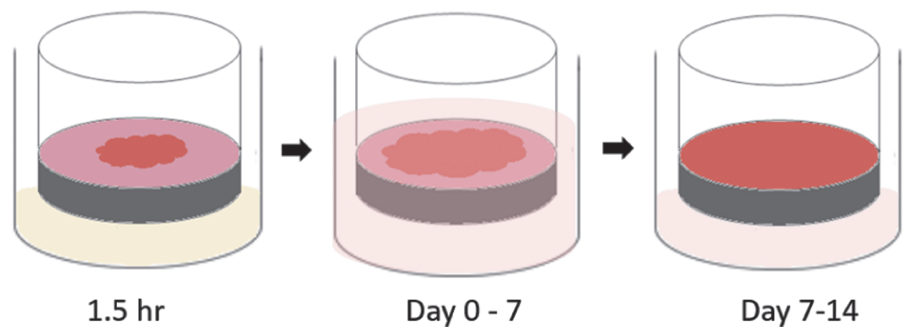

After $1.5 \mathrm{~h}$ incubation at RT, the constructs were submerged in epidermalization medium ${ }^{11}$ and placed in the incubator.

\section{Incubation/media protocol}

Epidermalization medium and cornification medium were formulated as described. ${ }^{11}$ Epidermalization medium was modified by the addition of aprotinin $(0.025 \mathrm{mg} / \mathrm{mL}$; Sigma A4529). The tissues were incubated $\left(37^{\circ} \mathrm{C}, 5 \% \mathrm{CO}_{2}\right)$ for 1 week submerged in the epidermalization medium. On day 7 , the tissues were transferred to deep-well plates (Greiner $665110)$ with media level adjusted to air-liquid interface and placed back in the incubator for another week in the cornification medium. Media were changed every 2 days throughout the 2-week incubation. On day 14, tissues were either fixed in 10\% Neutral Buffered Formalin or used for validation assays. Fixed tissues were embedded in paraffin and sliced for Hematoxylin and Eosin (H\&E) staining or immunohistochemistry (IHC).

\section{4-well format}

Bioprinted dermis region thickness in 24-well format (Corning 3450) was reduced to four layers to reduce concavity of the SE surface due to central vertical contraction after printing. Laminin/entactin and keratinocyte layers were miniaturized proportionally with respect to surface area.

The two protocols for pipetted samples differed in their dermal hydrogel formulation, with hydrogel comprising either DBH mixture or a collagen I plug $(4 \mathrm{mg} / \mathrm{mL}$; Corning $354249)$. A volume of $54 \mu \mathrm{L}$ hydrogel/ $\mathrm{HDF}_{\mathrm{N}}(2 \mathrm{million} / \mathrm{mL})$ mixture was applied, matching approximately the volume of printed samples. The laminin/entactin layer was applied by hand to DBH samples in 24 well, and keratinocyte suspension was manually dropped centrally on top of the construct.

\section{Histology}

BPSEs were fixed in $10 \%$ formalin overnight at $4^{\circ} \mathrm{C}$, then stored in $70 \%$ ethanol until ready for processing. Human postmortem samples were obtained from Tissue4research.com. Tissues were processed using the standard processing procedure on the ThermoFisher Excelsior AS Tissue Processor. Tissues were embedded in paraffin wax using the ThermoFisher HistoStar Embedding Workstation. Six-micrometer sections were cut on the ThermoFisher HM 355S Microtome and placed on positively charged slides. H\&E staining was performed on the ThermoFisher Gemini stainer using the predefined H\&E protocol. IHC was performed on the Leica Bond RXm using the predefined "Standard Protocol F" and the Bond Polymer Refine Detection Kit for DAB staining (Leica DS9800) with the following primary antibodies: COL-1 (Invitrogen PA126204), COL-VII (abcam ab6312), Ki67 (Leica PA0118), CK-1 (BioLegend 905201), FLG01 (Invitrogen MA5-13440), CK-15 (abcam ab52816), ZO-1 (Invitrogen 61-7300), CLD1 (abcam ab211737), and CDH1 (abcam ab1416). Slide images were taken with the Leica Aperio Versa 200.

\section{Imaging flattened Kelvin's tetrakaidecahedron}

Tissues were fixed at day 14 with $4 \%$ paraformaldehyde overnight, and subsequently preserved in PBS until use. To stain for cell membranes, the sample was incubated in phalloidin (ThermoFisher A34055) for 7 days. The transwell membrane was cut from the plastic support and inverted onto a glass coverslip for measurement on the Leica TCS SP8 with $40 \times$ objective.

3D optical coherence tomography imaging. A customized Spectral-Domain optical coherence tomography $(\mathrm{OCT})^{18}$ with superluminescent diode (cBLMD-T-850-HP; Superlum) provided broadband light with $850 \mathrm{~nm}$ central wavelength. A $4 \times$ objective (UPlanFl N $4 \times / 0.13$; Olympus) 
was used to achieve $\sim 8 \mu \mathrm{m}$ transverse resolution. The interference signal was detected by a spectrometer (Cobra-S 800; Wasatch) and datasets obtained using LabView. To image full well BPSEs ( $\sim 11 \mathrm{~mm}$ diameter), multiple data patches with overlap of $33 \%$ were flattened and stitched with Image ${ }^{19}$ and MATLAB. A 3D overview was created using Amira and Imaris and thicknesses assessed by ImageJ.

\section{3-(4,5-dimethylthiazol-2-yl)-2,5-diphenyltetrazolium bromide cell viability assay}

The assay was based on the published protocol provided by MatTek Corporation, Ashland, MA (In Vitro EpiDerm ${ }^{\mathrm{TM}}$ Skin Corrosion Test EPI-200-SCT $07 / 11 / 2014)$. Mature BPSEs were topically exposed to $10 \mu \mathrm{L} 5 \%$ sodium dodecyl sulfate (SDS) or water for $1 \mathrm{~h}$, and subsequently rinsed with PBS, blotted dry, and placed in fresh medium for a 2-day incubation. The medium was then replaced with 3-(4,5-dimethylthiazol-2yl)-2,5-diphenyltetrazolium bromide (MTT) (ATCC 30-1010K, $30 \mu \mathrm{L} / 270 \mu \mathrm{L})$ in fresh medium, and incubated $4 \mathrm{~h}$. Samples were then submerged in $2 \mathrm{~mL}$ isopropanol overnight at $4^{\circ} \mathrm{C}$. An optical density (OD) of $570 \mathrm{~nm}$ was measured on the PerkinElmer EnVision Plate Reader and data blank corrected with isopropanol.

\section{Permeability tracing with Lucifer yellow and biotin tracer}

To examine "outside in" physical barrier function, $15 \mu \mathrm{L}$ Lucifer yellow (LY) (1 mM; ThermoFisher L453) was pipetted to the top of the tissue at days 3 and 14 and incubated at $37^{\circ} \mathrm{C}$ overnight. Tight junction (TJ) "inside out" function was tested by replacing the medium with $3 \mathrm{~mL} 1.67 \mathrm{mg} / \mathrm{mL}$ biotin tracer (ThermoFisher 21335) on day 14 and incubated overnight. Tissues were fixed in $10 \%$ neutral buffered formalin, paraffin embedded, and dewaxed on slides. Biotin tracer slides were subsequently incubated 30 min with 1:200 Alexa Fluor 594 streptavidin (ThermoFisher S11227). Coverslips were mounted with DAPI Fluoromount-G ${ }^{\mathrm{TM}}$ (ThermoFisher 00-4959-52).

\section{Results and Discussion}

\section{Printed architecture composition}

DBH composition for minimal lateral tissue contraction. A critical aspect of using 3D printing as a biofabri- cation technique is the choice of the hydrogel formulation used as a bioink; the hydrogel material has to have suitable rheological properties of viscosity, low shear stress and biocompatibility to provide the appropriate printability, scaffolding, extracellular material, and composition to support tissue structure. The cells should be able to use the hydrogel as a support to be viable and functional. In the DBH formulation described, gelatin is included to provide appropriate viscosity so that the tissue structure is printed firm. Collagen is added as an ECM component to enhance cell adhesion, maintain tissue thickness, and reduce the time needed for the tissue to mature. The method used to mix the ingredients is of high importance so that all components can be mixed homogeneously, and maintain adequate viscosity properties before and after printing. A previous study reported that the maximum concentration of collagen that could be mixed homogeneously with a gelatin-containing mixture was $0.82 \mathrm{mg} /$ mL collagen. ${ }^{20}$ However, by neutralizing the formulation after addition of collagen rather than before, we achieved a maximum concentration of $4 \mathrm{mg} / \mathrm{mL}$.

Tissue contraction is a common occurrence in the generation of traditional raft cultures. ${ }^{21,22}$ For this reason, many raft protocols include an incubation period of up to 7 days to allow for contraction to subside before applying the epidermal cells. ${ }^{11}$ The method presented in this study minimizes lateral contraction (Fig. 2) by strengthening the gelatin/collagen matrix with fibrin, thus eliminating the need for a long incubation time before the application of epidermal cells. ${ }^{23}$ Streamlining the procedure in this way saves significant time, reduces consumption of medium during the development phase, and facilitates adaptability to a format with smaller diameter wells. With skin raft production methods devoid of fibrin, creation of tissues in smaller well formats tends to contract to the extent that they are unusably tiny and often drift to the edges of the well. By adding fibrin to the hydrogel, it allows for uniform flat tissues to form and help maintain the original diameter of the tissue design. It is worth noting that it was found to be important to bioprint the skin tissue to a diameter close to the well diameter so that the tissue can anchor to the well walls. Earlier attempts to print with a diameter smaller than the well size resulted in lateral contraction on the face while the base of the tissue maintained its diameter, forming a truncated cone shape (data
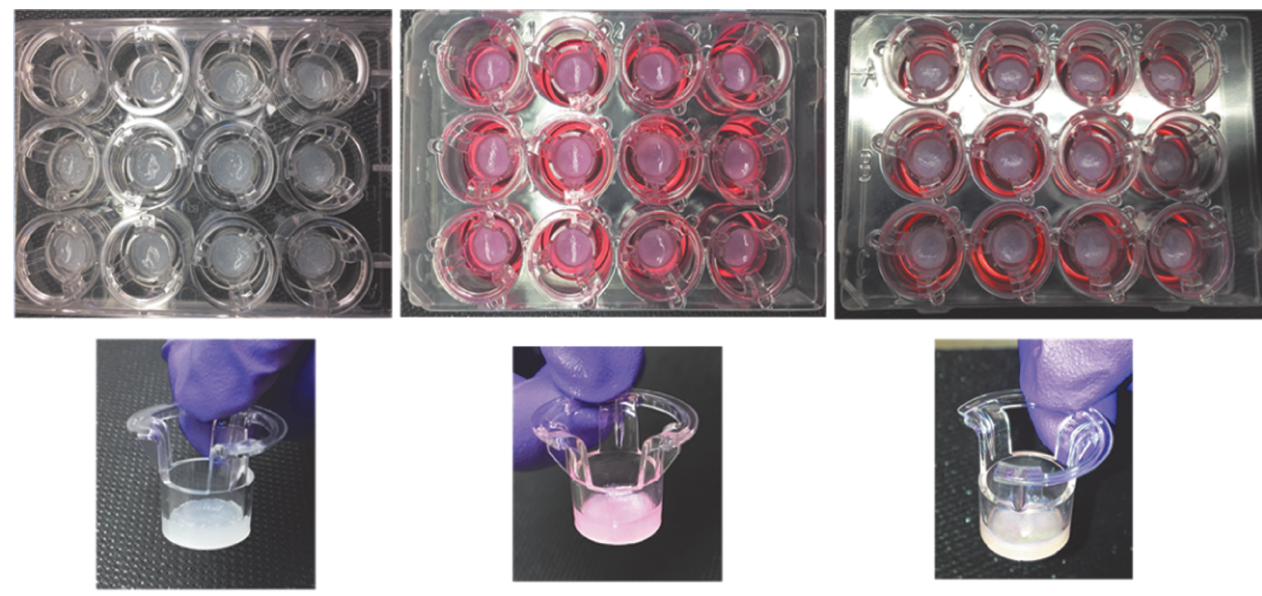

Day 0

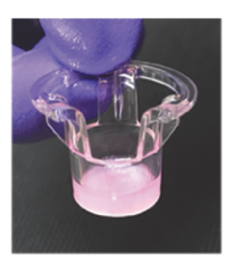

Day 7

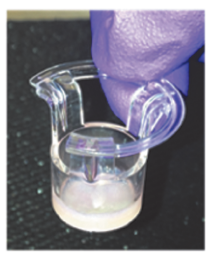

Day 12
FIG. 2. Minimal lateral contraction is observed as tissue matures. Full plate and single-well photos show that tissue maintains full well diameter throughout the time of tissue maturation. 
not shown). If smaller tissues are desired, this effect can be minimized by pipetting fibrinogen around the outside of the tissue to provide an anchor to the walls.

Vertical tissue contraction is not prevented with this method. Based on histological slices, the final thickness of the dermis is roughly $1 / 10$ of the original printed thickness of $2 \mathrm{~mm}$, thus yielding a final product with adequate dermal thickness for histological handling and analysis.

Laminin/entactin basal layer. The purpose of adding a laminin/entactin layer is threefold: It is an ECM component naturally found in the basement membrane, it is readily adherent for cells to attach, and it is believed to plug any gaps on the surface of the structure so that keratinocytes do not drift into the dermis before forming the stratum basale. Early attempts to build the construct without a laminin/ entactin layer and with a higher gelatin concentration resulted in occasional keratinocyte tumor-like formation within the dermis, possibly due to individual cells falling into the construct and growing underneath the basal monolayer.

Application of epidermal cells. As it is critical to epidermal formation that the stratum basal form a confluent monolayer, $\mathrm{NHEK}_{\mathrm{N}}$ are added in a small volume placed in the center of the construct as previously described, ${ }^{11}$ thus ensuring that the central region of the epidermis reaches confluency and may gradually extend to the edges.

Time to print. To reduce printing time, rather than successively print each tissue in its entirety, the dermal region was printed across the entire 12-well plate before printing each of the two subsequent regions. This reduced the incidence of print head changes throughout the process. Timing required for dermal, basal, and epidermal regions was 18:09, 5:20, and 2:00 (in minutes:seconds), respectively. Allowing for $1 \mathrm{~min}$ between successive layers for a user to load and execute the subsequent G-code, printing time is $2.5 \mathrm{~min} /$ tissue.

\section{Structural analysis of the $3 D$ BPSEs}

Histological analysis. H\&E staining of a paraffinembedded slice of native human skin compared with the BPSE (Fig. 3a) shows that the BPSE appears morphologically similar to normal human skin, with the four primary layers of the epidermis represented. The dermis appears uniform, with no evidence of individual printed layers within the dermis, possibly attributed to collagen deposition and rearrangement by the cells within the dermis as the construct matures. ${ }^{24}$

Evaluation of cell density in native human dermis has been determined to be between 2000 and 4000 cells $/ \mathrm{mm}^{3}{ }^{25}$ To create morphologically similar tissues, a fibroblast concentration of $2000 / \mathrm{mm}^{3}$ in the DBH mixture was used. Cell density of mature BPSEs was evaluated using one representative sample from each of four batches (Fig. 3b). Tissue slides were dewaxed and stained with DAPI. Dermal cell density was counted within a $1500 \mu \mathrm{m}^{2}$ sample area, and compared with native human samples. Evaluation of dermal cell density in mature BPSEs was determined to be $14,000 \pm 4000$ cells $/ \mathrm{mm}^{2}$, and that of human samples was $21,000 \pm 7000$ cells $/ \mathrm{mm}^{2}$.

The 3,3'-diaminobenzidine (DAB) staining immunohistochemistry confirms localization of epidermal differentiation and proliferation markers, tight junction markers, as well as ECM proteins. Figure 4 shows the presence of TJ proteins ZO-1, Claudin I, ${ }^{26}$ and E Cadherin ${ }^{27}$ with expression localization comparable to human skin. Likewise, epidermal differentiation markers, Keratin 1, Filaggrin, and Keratin 15 are shown as well as ECM protein markers Collagen I and Collagen VII, and proliferation marker Ki67.

Tissue viability-MTT assay. A commonly used cell viability assay, the colorimetric yellow tetrazolium MTT conversion assay has been established as an effective test to screen for topical irritants on skin tissue samples ex vivo and in vitro. ${ }^{28,29}$ To gauge tissue viability (TV) and demonstrate suitability of BPSEs for bioassay utilization, the MTT assay was performed on a plate of tissues at 14 days of age. The assay was performed as described in the published protocol (In Vitro EpiDerm Skin Corrosion Test EPI-200-SCT $07 / 11 /$ 2014; MatTek, Ashland, MA). BPSEs were exposed to $5 \%$
FIG. 3. Histological comparison of BPSE versus native human skin. (a) H\&E staining shows clear layers of differentiated keratinocytes. (b) Dermal cell density of BPSE and human skin was estimated by counting number of nuclei within a $1500 \mu \mathrm{m}^{2}$ sample area from four representative samples of each tissue type. Density $_{\text {BPSE }}=14,000 \pm 4000$ cells/ $\mathrm{mm}^{2}$, Density Human $_{2}=21,000$ \pm 7000 cells $/ \mathrm{mm}^{2}$. Scale bars, $100 \mu \mathrm{m}$. H\&E, Hematoxylin and Eosin. a

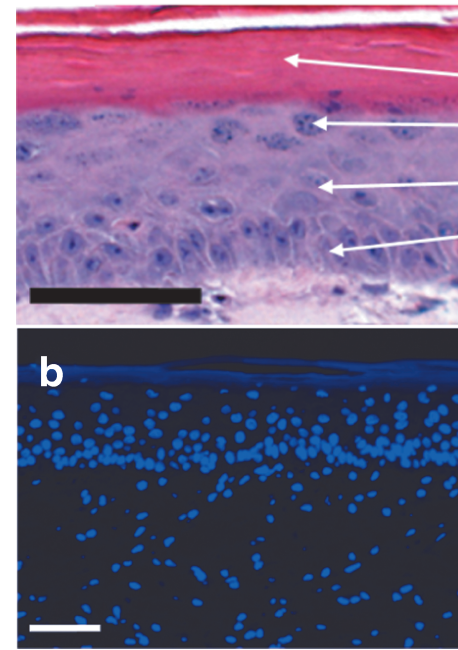

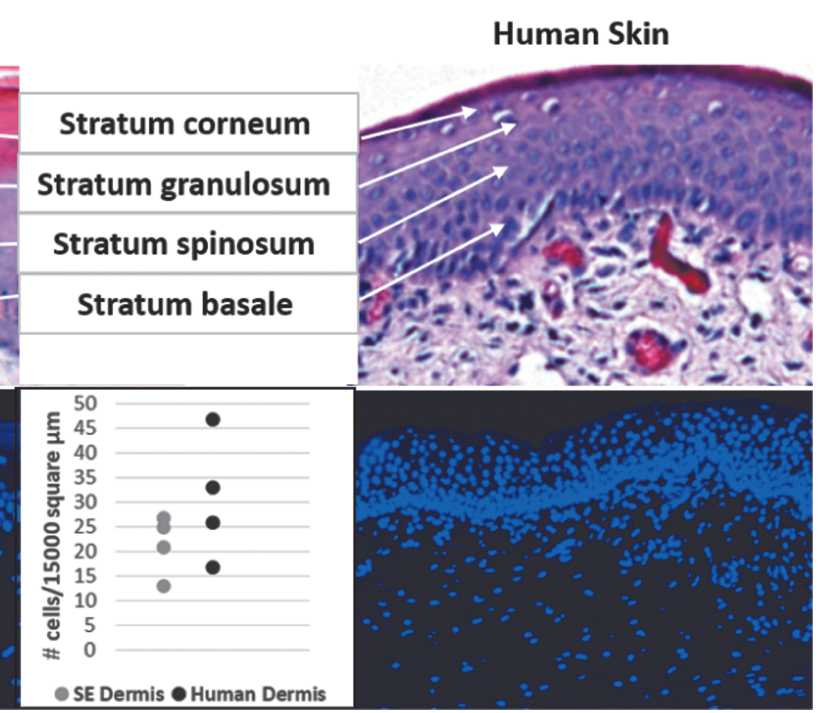



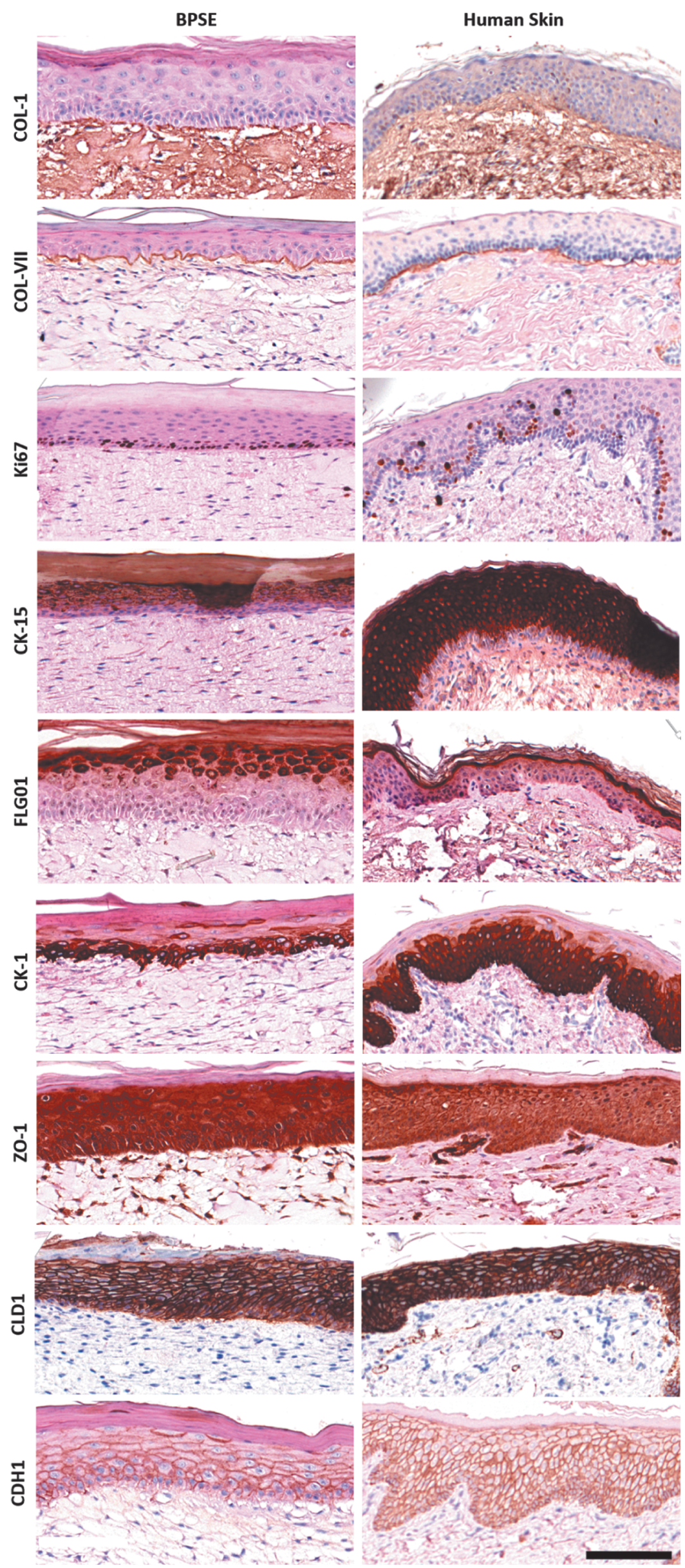

FIG. 4. IHC comparison of protein expression between bioprinted tissues and native human skin show comparable morphology. Tight junction proteins ZO-1, Claudin I, and E-Cadherin. Epidermal differentiation proteins Keratin 1, Filaggrin, and Cytokeratin 15. ECM proteins Collagen I and Collagen VII and cell proliferation marker Ki67. Scale bar, $100 \mu \mathrm{m}$.

ECM, extracellular matrix;

IHC, immunohistochemistry. 


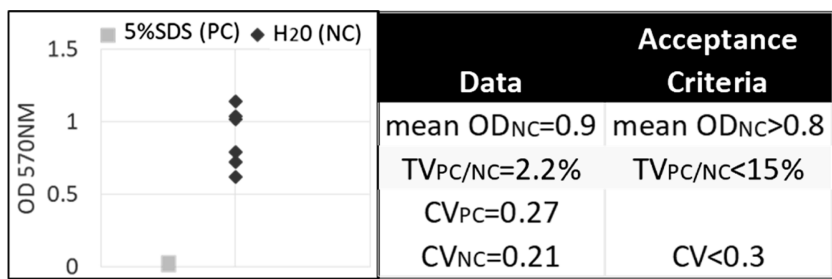

FIG. 5. MTT cell viability measurements were taken 2 days after exposure to 5\% SDS as PC or water as NC. Mean Optical Density ${ }_{\mathrm{NC}}\left(\mathrm{OD}_{\mathrm{NC}}\right)$, Ratio of Tissue Viability $\mathrm{PC}_{\mathrm{NC}}$ $\left(\mathrm{TV}_{\mathrm{PC} / \mathrm{NC}}\right)$ and $\mathrm{CV}$ were within Acceptance Criteria. $n=6$. $\mathrm{CV}$, coefficients of variation; MTT, 3-(4,5-dimethylthiazol-2yl)-2,5-diphenyltetrazolium bromide; NC, negative control; PC, positive control; SDS, sodium dodecyl sulfate.

SDS, a known irritant, as a positive control (PC), and water as a negative control (NC). Two days after exposure, absorbance readings were taken at $570 \mathrm{~nm}$, with larger data values corresponding to higher cell viability. As seen in Figure 5, mean optical density $_{\mathrm{NC}}(\mathrm{OD})$, ratio of tissue viability PC/NC $_{\text {(TV) }}$ and coefficients of variation were within MatTek's Acceptance Criteria ( $>0.8,<15 \%$, and $<0.3$, respectively) showing low well to well variability between replicates.

Flattened Kelvin's tetrakaidecahedron shape of the stratum granulosum. To maintain barrier function, TJs are formed by differentiated keratinocytes in the stratum granulosum layer of the skin. It has been shown that at the stratum granulosum layer, differentiated keratinocytes adopt a flattened Kelvin's tetrakaidecahedron (f-TKD) shape $^{5}$ allowing the cells to achieve homeostasis of the TJ barrier (Fig. 6a). f-TKD morphology are observed in fixed tissues by staining with a probe such as phalloidin that enables visualization of cell membrane morphology. f-TKDs appear as double-edged polygons when viewed with fluorescence microscopy.

3D nondestructive imaging of BPSEs by OCT. OCT is an emerging 3D imaging modality that scans with nearinfrared light and detects back-scattering light from different layers of tissue. ${ }^{30}$ OCT has been widely used in ophthalmology and dermatology due to its high speed and nondestructive imaging capability. ${ }^{31-33}$ Live OCT imaging of BPSEs (Fig. 6b-d) indicates a uniform tissue with extensive epidermal coverage. Full well reconstruction reveals a concavity indicating greater vertical contraction away from the well walls. Layer depths are measured at various positions to illustrate the potential of the technology for quality control of live samples in real time.

\section{Barrier function}

Barrier function of the skin, facilitated by TJs, is the primary function of the organ in the body. ${ }^{26,34}$ Barrier function was validated through penetration assays and electrical impedance as an assessment of the integrity of the TJs.

Electrical conductivity. The relationship between hydration of the epidermis and electrical conductivity of skin surface has been well studied. ${ }^{35}$ The magnitude of electrical impedance on the surface of the skin correlates to the formation of an effective epidermal permeability barrier ${ }^{36-38}$ ). Electrical conductivity across the dermal surface can be measured using a Nova Dermal Phase Meter 9003 (Nova Technologies) with data reported in Dermal Phase Meter (DPM) arbitrary units and values increasing with higher conductivity. ${ }^{36,37}$ Previous studies have shown that native human skin has conductivity ranging from $\sim 100$ to 168 $\mathrm{DPM}^{38,39}$ and untreated EpiDerm engineered SE (MatTek) was measured to be $\sim 100 \mathrm{DPM}^{40}$ Measurements of 12 BPSEs from a single batch yielded conductivity values of $117 \pm 10$ DPM, within the range of measured human dermis. As a NC eight printed dermis-only (no epidermis) samples gave a value of $831 \pm 19$ DPM (Fig. 7a).

Penetration assay. Barrier function was further assessed by performing biotin tracer and LY penetration assays, as described previously. ${ }^{41}$ Barrier integrity "inside out" function was tested with biotin tracer dissolved into tissue media and allowed to diffuse through the tissue from beneath. Figure $7 \mathrm{~b}$
FIG. 6. Structural Analysis of BPSEs. (a) Confocal imaging of cells in stratum granulosum of BPSE shows double-sided hexagons indicative of $f-T K D$ shape representing TJ formation and allowing the cells to achieve homeostasis of the TJ barrier. Scale bar, $10 \mu \mathrm{m}$. (b-d) Optical coherence tomography image of BPSE shows tissue uniformity. (b) $3 \times 3 \mathrm{~mm}^{2}$ reconstructed section shows uniform distinct tissue layers. (c) Full diameter $(\sim 11 \mathrm{~mm})$ tissue image cross-section and 3D full rendering show extensive epidermal coverage and 3D concave shape. (d) Layer thicknesses are measured for stratum corneum (i), live epidermal layers (ii), and dermis (iii). Scale bar, $200 \mu \mathrm{m}$. 3D, three-dimensional; f-TKD, flattened Kelvin's tetrakaidecahedron; TJ, tight junction.

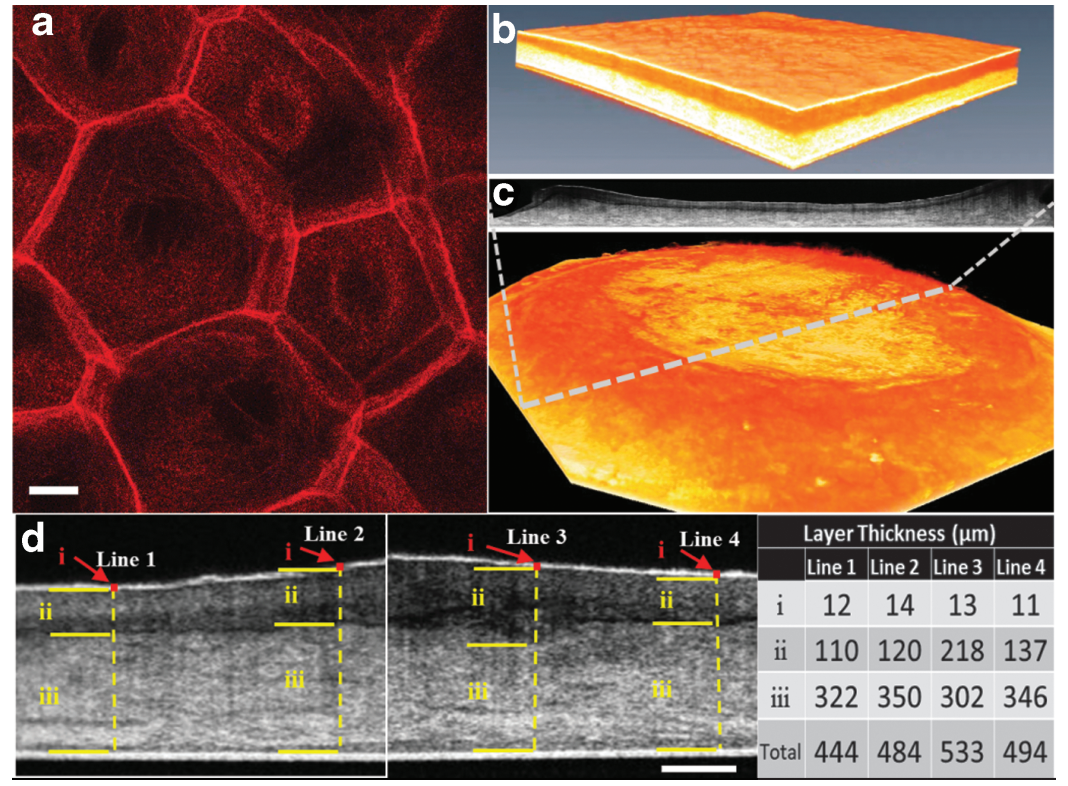



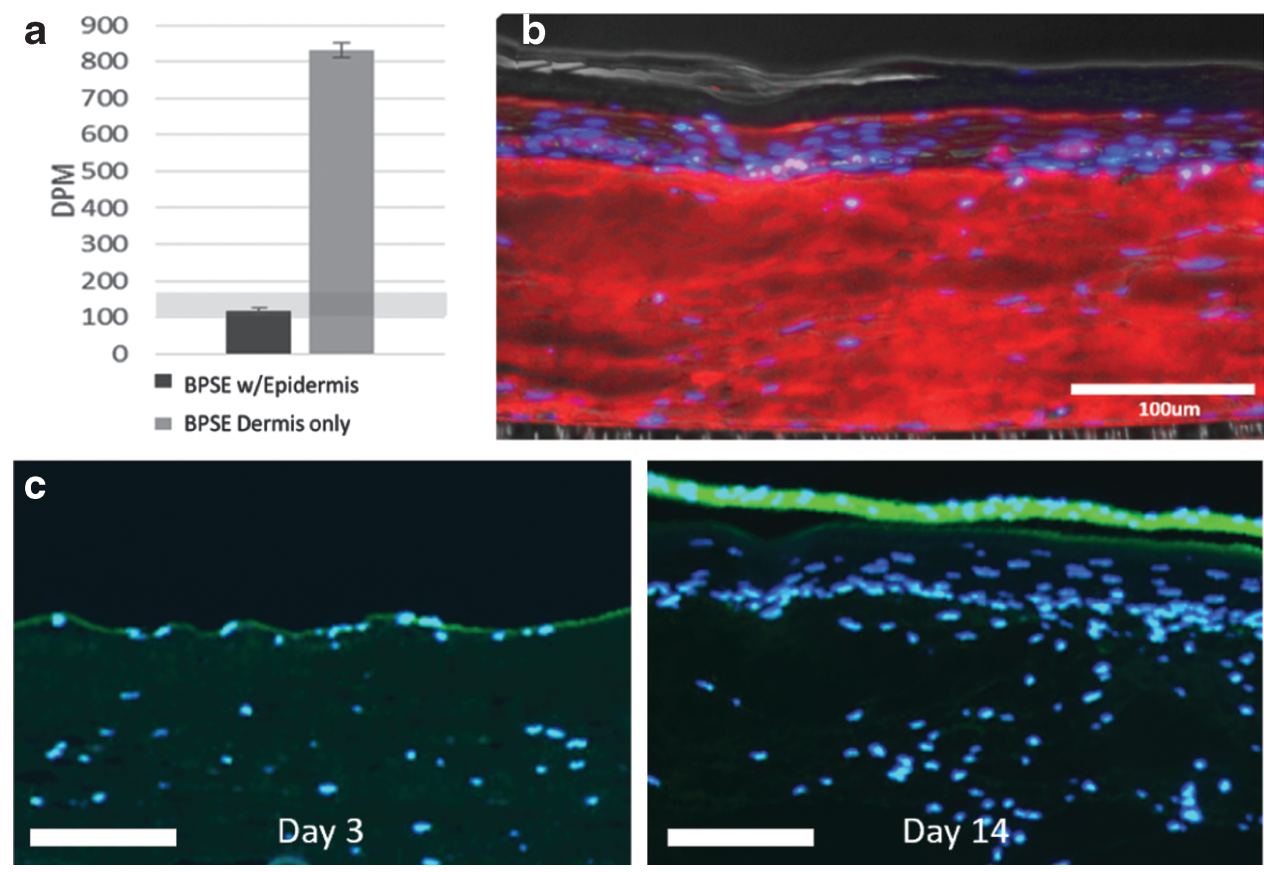

FIG. 7. Barrier Function Assessment. (a) Electrical conductivity measurement across surface of BPSE. DPM units are arbitrary. Conductivity of SE with epidermis $(117 \pm 10 \mathrm{DPM}$, $n=12$ ) is within range of previously reported measurements of native human skin (100 to 168 DPM). BPSE lacking epidermal layer measure conductivity of $831 \pm 19$ DPM; $n=8$. (b) "Inside out" Barrier Function Penetration Assay: Biotin tracer ( $r e d$ ) diffused from beneath the tissue does not reach the cornified epidermis. (c) "Outside in" Barrier Function Penetration Assay: Lucifer yellow (green)

placed on top of the tissue at days 3 and 14. Scale bar, $100 \mu \mathrm{m}$. DPM, Dermal Phase Meter; SE, skin equivalent. shows that the tracer is able to penetrate through the tissue up to the depth of the TJ. To gauge "outside in" physical barrier function, LY fluorescent dye was placed on the surface of the epidermis on day 14 . As a $\mathrm{NC}$, the assay was performed on tissues at day 3, before formation of epidermal TJs. Figure 7c indicates accumulation of LY dye on top of the tissue when performed at day 14, and diffusion into the construct on day 3 .

\section{Higher throughput well format}

To examine the utility of the protocol for smaller well formats, experiments were performed to compare the described bioprinted protocol to pipetted protocols. Tissues were bioprinted into 24-well transwells and compared with two separate pipetted protocols, with dermal region comprising $\mathrm{HDF}_{\mathrm{N}}$ in either DBH or a collagen I plug as in traditional skin raft cultures. ${ }^{42}$

Lateral contraction for printed and pipetted tissues appeared to be minimal for samples made with $\mathrm{DBH}$, while collagen I samples contracted significantly to an extent that histological analysis was not feasible (Supplementary Fig. S1). Measurement of transepithelial electrical resistance indicates higher barrier integrity in printed samples versus pipetted with $\mathrm{DBH}$. $\mathrm{H} \& \mathrm{E}$ staining of histology slices indicates more distinct epidermal differentiation in printed samples over pipetted. Furthermore, pipetted samples exhibited uneven dermal thickness with a thin central region, likely due to meniscus formation before gelation of dermal layer.

\section{Discussion}

This study demonstrates biofabrication of morphologically and physiologically relevant BPSEs in a multiwell plate format. The constructs were structurally validated by multiple IHC markers. Impedance measurement, penetration assays, and cell viability assays show viable tissues with good barrier function and well-to-well reproducibility. The constructs are fully bioprinted on an open-market printer with easy-to-use software for designing architecture in a layer-by-layer model. The ability to BPSEs in a multiwellbased platform should enable the robust testing of compounds for toxicity effects and the development of skin disease models for drug testing. In this regard, stressors or diseased cells can be introduced and their phenotypic effects on the native state measured using quantitative detection methods commonly used in HTS. The addition of vasculature, immune cells, and disease conditions will make these tissue equivalents even more relevant to study skin diseases. In addition, the use of OCT measurements in this work illustrates how noninvasive and nondestructive imaging technologies already clinically used can be applied to biofabricated tissues to quantitatively assess the correct structure and serve as quality control technology during tissue production. In our experience, the use of primary keratinocytes still remains a challenge in terms of obtaining consistent results during production, as growth rate and differentiation capacity can differ due to lot variability. It is the hope that the development of induced pluripotent stem cell-differentiation protocols for the production of keratinocytes will help obtain more consistent cells and consequently tissues.

Furthermore, development of disease models such as Squamous Cell Carcinoma and Psoriasis, as well as more complex models incorporating features such as innervation will provide avenues to explore advanced drug screening for disease states.

\section{Acknowledgments}

The authors would like to thank Qiongyu Guo and Yongyang Huang from Lehigh University for providing assistance with early skin imaging protocols and data acquisition. This project was supported by the National Institutes of Health Intramural Research Program, the Cures Acceleration Network program at the National Center for Advancing 
Translational Sciences, NSF grant IDBR 1455613, and NIH grant R01EB025209.

\section{Disclosure Statement}

No competing financial interests exist.

\section{Supplementary Material}

Supplementary Figure S1

\section{References}

1. Santoro, M., Navarro, J., and Fisher, J.P. Micro- and macrobioprinting: current trends in tissue modeling and organ fabrication. Small Methods 1700318, 1, 2018.

2. Ma, X., Liu, J., Zhu, W., et al. 3D bioprinting of functional tissue models for personalized drug screening and in vitro disease modeling. Adv Drug Deliv Rev 132, 235, 2018.

3. Bouwstra, J.A. The skin barrier, a well-organized membrane. Colloids Surfaces 124, 403, 1997.

4. Elias, P.M. Skin barrier function. Curr Allergy Asthma Rep 8, 299, 2008.

5. Yokouchi, M., Atsugi, T., Van Logtestijn, M., et al. Epidermal cell turnover across tight junctions based on Kelvin's tetrakaidecahedron cell shape. Elife Elsevier Inc 5, 1, 2016.

6. Wickett, R.R., and Visscher, M.O. Structure and function of the epidermal barrier. Am J Infect Control 34, 98, 2006.

7. Andrade, T.A., Aguiar, A.F., Guedes, F.A., et al. Ex vivo model of human skin (hOSEC) as alternative to animal use for cosmetic tests. Procedia Eng Elsevier BV 110, 67, 2015.

8. De Wever, B., Kurdykowski, S., and Descargues, P. Human skin models for research applications in pharmacology and toxicology: introducing NativeSkin ${ }^{\circledR}$, the "Missing Link" bridging cell culture and/or reconstructed skin models and human clinical testing. Appl In Vitro Toxicol 1, 26, 2015.

9. Dumont, C., Prieto, P., Asturiol, D., and Worth, A. Review of the availability of in vitro and in silico methods for assessing dermal bioavailability. Appl In Vitro Toxicol 1, 147, 2015.

10. Anacker, D., and Moody, C. Generation of organotypic raft cultures from primary human keratinocytes. J Vis Exp 3668, 2012.

11. Gangatirkar, P., Paquet-Fifield, S., Li, A., Rossi, R., and Kaur, P. Establishment of 3D organotypic cultures using human neonatal epidermal cells. Nat Protoc 2, 178, 2007.

12. Cubo, N., Garcia, M., Del Cañizo, J.F., Velasco, D., and Jorcano, J.L. 3D bioprinting of functional human skin: production and in vivo analysis. Biofabrication 9, 1, 2017.

13. Koch, L., Deiwick, A., Schlie, S., et al. Skin tissue generation by laser cell printing. Biotechnol Bioeng 109, 1855, 2012.

14. Min, D., Lee, W., Bae, I.H., Lee, T.R., Croce, P., and Yoo, S.S. Bioprinting of biomimetic skin containing melanocytes. Exp Dermatol 27, 453, 2018.

15. Pourchet, L.J., Thepot, A., Albouy, M., et al. Human skin 3D bioprinting using scaffold-free approach. Adv Healthc Mater 6, 1, 2017.

16. Lee, V., Singh, G., Trasatti, J.P., et al. Design and fabrication of human skin by three-dimensional bioprinting. Tissue Eng Part C Methods 20, 473, 2014.

17. Ng, W.L., Qi, J.T.Z., Yeong, W.Y., and Naing, M.W. Proof-of-concept: 3D bioprinting of pigmented human skin constructs. Biofabrication 10, 025005, 2018.
18. Li, F., Song, Y., Dryer, A., Cogguillo, W., Berdichevsky, Y., and Zhou, C. Nondestructive evaluation of progressive neuronal changes in organotypic rat hippocampal slice cultures using ultrahigh-resolution optical coherence microscopy. Neurophotonics 1, 025002, 2014.

19. Schindelin, J., Arganda-Carreras, I., Frise, E., et al. Fiji: an open source platform for biological image analysis. Nat Methods 9, 676, 2012.

20. Wu, Z., Su, X., Xu, Y., Kong, B., Sun, W., and Mi, S. Bioprinting three-dimensional cell-laden tissue constructs with controllable degradation. Sci Rep 6, 1, 2016.

21. Bell, E., Ivarsson, B., and Merrill, C. Production of a tissuelike structure by contraction of collagen lattices by human fibroblasts of different proliferative potential in vitro. Proc Natl Acad Sci U S A 76, 1274, 1979.

22. Rompre, P., Auger, F.A., Germain, L., et al. Influence of initial collagen and cellular concentrations on the final surface area of dermal and skin equivalents: a BoxBehnken analysis. Vitr Cell Dev Biol 26, 983, 1990.

23. Gsib, O., Egles, C., and Bencherif, S.A. Fibrin: an underrated biopolymer for skin tissue engineering. J Mol Biol Biotechnol 2, 1, 2017.

24. Guidry, C., and Grinnell, F. Studies on the mechanism of hydrated collagen gel reorganization by human skin fibroblasts. J Cell Sci 79, 67, 1985.

25. Helary, C., Foucault-Bertaud, A., Godeau, G., Coulomb, B., and Giraud Guille, M.M. Fibroblast populated dense collagen matrices: cell migration, cell density and metalloproteinases expression. Biomaterials 26, 1533, 2005.

26. Brandner, J.M., Kief, S., Wladykowski, E., Houdek, P., and Moll, I. Tight junction proteins in the skin. Skin Pharmacol Physiol 19, 71, 2006.

27. Tunggal, J.A., Helfrich, I., Schmitz, A., et al. E-cadherin is essential for in vivo epidermal barrier function by regulating tight junctions. EMBO J 24, 1146, 2005.

28. Castagnoli, C., Alotto, D., Cambieri, I., et al. Evaluation of donor skin viability: fresh and cryopreserved skin using tetrazolioum salt assay. Burns 29, 759, 2003.

29. Watanabe, T., Hasegawa, T., Takahashi, H., Ishibashi, T., Itagaki, H., and Sugibayashi, K. Utility of MTT assay in three-dimensional cultured human skin model as an alternative for Draize skin irritation test: approach using diffusion law of irritant in skin and toxicokinetics-toxicodynamics correlation. Pharm Res 19, 669, 2002.

30. Huang, D., Swanson, E.A., Lin, C.P., et al. Optical coherence tomography. Science 80, 1178, 1991.

31. Geitzenauer, W., Hitzenberger, C.K., and Schmidt-Erfurth, U.M. Retinal optical coherence tomography: past, present and future perspectives. Br J Ophthalmol 95, 171, 2011.

32. Olsen, J., Holmes, J., and Jemec, G.B.E. Advances in optical coherence tomography in dermatology - a review. J Biomed Opt 23, 1, 2018.

33. Gambichler, T., Jaedicke, V., and Terras, S. Optical coherence tomography in dermatology: technical and clinical aspects. Arch Dermatol Res 303, 457, 2011.

34. Madison, K.C. Barrier function of the skin: "La Raison d'Être" of the epidermis. J Invest Dermatol Elsevier Masson SAS 121, 231, 2003.

35. Blichmann, C., and Serup, J. Assessment of skin moisture. Measurement of electrical conductance, capacitance and transepidermal water loss. Acta Derm Venereol 68, 284, 1998.

36. Boyce, S.T., Supp A.P., Harriger, M.D., Pickens, W.L., Wickett, R.R., and Hoath, S.B. Surface electrical capaci- 
tance as a noninvasive index of epidermal barrier in cultured skin substitutes in athymic mice. J Invest Dermatol 107, 82, 1996.

37. Goretsky, M.J., Supp, A.P., Greenhalgh, D.G., Warden, G.D., and Boyce, S.T. Surface electrical capacitance as an index of epidermal barrier properties of composite skin substitutes and skin autografts. Wound Repair Regen 3, 419, 1995.

38. Clarys, P., Barel, A.O., and Gabard, B. Non-invasive electrical measurements for the evaluation of the hydration state of the skin: comparison between threeconventional instruments- the Comeometer@, the Skicon@and the Nova DPM@. Skin Res Technol 5, 14, 1999.

39. Centanni, J.M., Straseski, J.A., Wicks, A., et al. Stratagraft skin substitute is well-tolerated and is not acutely immunogenic in patients with traumatic wounds: results from a prospective, randomized, controlled dose escalation trial. Ann Surg 253, 672, 2011.

40. Mei Xiong, Z., O’Donovan, M., Sun, L., Young Choi, J., Ren, M., and Cao, K. Anti-aging potentials of methylene blue for human skin longevity. Sci Rep 7, 1, 2017.
41. Smits, J.P.H., Niehues, H., Rikken, G., et al. Immortalized N/TERT keratinocytes as an alternative cell source in 3D human epidermal models. Sci Rep 7, 1, 2017.

42. Simpson, C.L., Kojima, S., and Getsios, S. RNA interference in keratinocytes and an organotypic model of human epidermis. Methods Mol Biol 585, 127, 2010.

Address correspondence to: Paige Derr, PhD

Department of Pre-clinical Innovation National Center for Advancing Translational Sciences National Institutes of Health Rockville, MD 20850

E-mail: paige.derr@nih.gov

Received: November 13, 2018 Accepted: April 16, 2019 Online Publication Date: June 12, 2019 